\title{
Vivre mieux aujourd'hui
}

\author{
Jean Martin
}

Dr med., membre de la rédaction

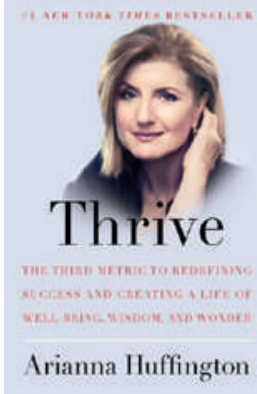

Arianna Huffington

Thrive

The Third Metric to Redefining Success and Creating a Life of Well-Being, Wisdom, and Wonder

New York: Harmony Books / Penguin Random House; 2015.

342 pages. $19.90 \mathrm{CHF}$.

ISBN 978-0-8041-4086-7

Arianna Huffington est née à Athènes en 1950. Elle a fait des études en Grande-Bretagne dès l'âge de 16 ans. Plus tard, elle a émigré aux Etats-Unis. A écrit une quinzaine de livres depuis 1973, y compris des biographies de Maria Callas et de Picasso. D'abord conservatrice, elle a pris dans les années 2000 une orientation libérale (au sens américain, à savoir progressiste) et s'est engagée en politique en Californie. Active aussi pour la cause des femmes. En 2005, elle est cofondatrice et rédactrice en chef d'un site web d'informations et commentaires politico-sociétaux, The Huffington Post, un des organes de presse électronique qui aujourd'hui retiennent le plus l'attention aux EtatsUnis. A participé à plusieurs WEF de Davos et on la trouve dans des listes de personnes les plus influentes. Son dernier ouvrage, Thrive (prospérer, se développer) est la présentation substantielle des leçons de vie d'une personne, hyperefficace et au sommet d'une carrière, qui prend du recul. Elle prend ses distances avec la culture US qui lui a pourtant apporté le succès. Sur la base de ses expériences personnelles, elle présente raisons et moyens de changements qu'elle croit nécessaires dans la manière de mener sa vie - au plan individuel aussi bien que collectif. Le but de son livre, dit-elle, est de «fix (réparer) our broken definition of success».

\section{Constat de départ - Succès professionnel et/vs vie personnelle réussie}

«De plus en plus de gens, de tous âges et milieux, en viennent à réaliser qu'il y a plus dans la vie que grimper les échelons hiérarchiques, que nous sommes plus que nos curricula vitae et que nous n'avons pas à accepter la notion que le burnout est le prix obligatoire du succès.» - «Des centaines de personnes m'ont dit com- bien leurs vies ont été transformées après tel incident marquant (wake-up call - par exemple un accident de santé), quand elles ont compris qu'elles ne consacraient pas assez de temps à ce qui vraiment avait de la valeur pour elles» (la traduction des citations est de J. M.).

Commentaire sur l'omniprésence des réseaux sociaux: «Dans le monde médiatique, la fétichisation des réseaux sociaux a atteint la proportion d'adoration des idoles. Conférences et congrès sont pleins de tables rondes à leur sujet et à la manière d'augmenter leur diffusion. Mais il est rare d'entendre quiconque discuter de la valeur de l'anecdote dont vous voudriez qu'elle inonde le monde.» - «Notre culture médiatique est bloquée sur le 〈Perpetual Now», la chasse aux scoops éphémères dont la plupart sont sans aucun intérêt.» «Notre surconnectivité est le serpent de notre Jardin d'Eden digital!»

Un mot sur la génération dite Y (ou «the millenials»): «Notre définition actuelle du succès et l'addiction à nos instruments électroniques est toxique et a un impact particulièrement négatif sur la nouvelle génération, dont des études importantes montrent qu'elle est la plus stressée parmi les classes d'âge.»

\section{La "Third Metric", son introduction dans la vie des gens et des entreprises}

Le grand thème du livre est le besoin d'ajouter aux deux metrics (mesures, indicateurs) usuelles du succès dans notre société, à savoir l'argent et le pouvoir, une troisième: comprenant quatre piliers caractérisés par les mots de bien-être, sagesse, capacité d'émerveillement (wonder) et pratique du don et du bénévolat (giving).

Un des buts de l'auteure, de façon plutôt convaincante, est de présenter des manières de limiter l'emprise sur nous de la pression du travail, évoquant les emplois du temps de ceux qui constamment courent à de multiples tâches (multitasking) - elle parle de "time famine». Les ayant expérimentés, elle propose divers moyens: méditation, comment refuser la surcharge de travail, dormir assez - elle est devenue une propagandiste du besoin, et de la règle, d'avoir au moins 7-8 heures de sommeil par nuit. Avec cela l'exercice physique bien sûr. Elle discute le "Slow Movement»: alimentation lente, manières lentes de voyager, de vivre, d'être parent, jardinage lent et même science lente. 


\section{Santé et travail, bien-être des collaborateurs}

Cheffe d'entreprises elle-même, elle est acquise à beaucoup plus d'attention à ce thème. Citation d'un professeur de Stanford: "Le manque d'attention aux besoins des employés contribue à expliquer pourquoi les EtatsUnis dépensent beaucoup plus que d'autres pays pour la santé, en fait la maladie, avec de moins bons résultats. J'espère que les entreprises vont se réveiller, prendre conscience du fait que, si elles ne s'occupent pas bien de leurs employés, elles-mêmes n'iront pas bien.» - «Un des principaux obstacles à l'amélioration souhaitable des conditions de travail est le mythe obstiné qu'il y a une ‘opposition` entre haute performance au travail et le fait de prendre soin de soi. Cette idée ne pourrait pas être plus fausse.»

Arianna Huffington donne des exemples de grandes firmes américaines qui ont mis en place des programmes permettant à leurs collaborateurs, sur le lieu de travail, de se détendre, méditer ou faire du yoga, prendre de l'exercice, voire de faire la sieste (nap-rooms!). Cela peut inclure des mesures contraignantes comme le blocage des instruments électroniques, qui empêche de travailler tard le soir ou le week-end. A propos de relations humaines: «Gérer de manière empathique (managing compassionately) implique de communiquer de manière transparente et de pratiquer «la marche dans les chaussures de l'autre: il est toujours constructif de prendre une minute pour comprendre pourquoi d'autres personnes sont arrivées à leur conclusion [différente]. Est-ce expliqué par des éléments de leur histoire?» Citation d'un ouvrage de H. Benson et W. Proctor, basé sur des données cliniques et épidémiologiques: «La même science que celle utilisée pour évaluer l'efficacité des médicaments et des techniques médicales montre que notre esprit peut guérir notre corps. Ce n'est pas une exagération de dire que presque tous les problèmes de santé peuvent être améliorés par une (mind-body approach), notamment la méditation. C'est le couteau suisse des moyens médicaux!» (sic). Ces auteurs veulent que cette approche soit vue comme la troisième grande option thérapeutique aux côtés de la chirurgie et des médicaments.

\section{Quelques points encore}

Le «giving» fait partie de la «Third Metric». Huffington y inclut l'empathie: «La «compassionate empathy» est un talent que nous pouvons nourrir, celui qui nous remplit quand nous donnons en retour. Mais le terme ‘donner en retour` peut être mal compris; en effet, il semble indiquer que le contact humain et le bénévolat importent seulement par ce que nous faisons pour le bénéficiaire ou la communauté. Mais, les données scientifiques à cet égard sont très claires, ces échanges sont tout autant au bénéfice de la personne qui aide. Donner est une sorte de médicament-miracle, sans aucun effet secondaire indésirable.»

On trouve une section sur la mort et notre attitude fuyante à son égard, où sont évoqués les philosophes antiques, les traditions religieuses, Elisabeth KüblerRoss ou tel moine bouddhiste. Arianna Huffington mentionne les "Cafés mortels» de Bernard Crettaz (dont elle sait qu'ils sont nés en Suisse) et «The Conversation Project", dont l'atout, dit Ellen Goodman, sa fondatrice, est que «c'est à leur table de cuisine et pas dans une unité de soins intensifs que nous proposons aux gens de parler. La différence entre une ‘bonne mort» et une «mort difficile» semble être que la personne ait eu la possibilité d'échanger sur ses vœux pour la fin de vie. Malheureusement, cet échange n'est pas fréquent, il y a conspiration du silence [...] Tous nous pensons souvent qu'il est trop tôt pour parler de la mort. Jusqu'à ce qu'il soit trop tard.»

\section{Perspectives}

Même si une hirondelle ne fait pas le printemps, Huffington affirme voir aujourd'hui que les options et modes de vie évoluent vite, pour le mieux. «Même dans le monde de la banque et de la finance, incubateurs de burnout, le passé récent a vu un changement considérable. Ces sujets ne sont plus considérés comme alternatifs ou «new ages, ils sont maintenant ancrés dans l'opinion générale.»

Thrive fourmille de références à la littérature, y compris travaux scientifiques dans les domaines psychologique, comportemental, sociologique, médical. Ce côté très fouillé n'empêche pas l'auteur de citer Nancy Koehn, professeure à Harvard: «Information n'est pas synonyme de connaissance, connaissance n'est pas synonyme de compréhension et compréhension n'est pas synonyme de sagesse - ce dont nous avons besoin, c'est de sagesse."

Les messages voire les enthousiasmes de ce livre peuvent donner l'impression de redire des choses déjà abondamment relevées aujourd'hui. A mon sens, ce que dit Huffington a un poids particulier au vu de sa trajectoire peu ordinaire. Elle a trouvé le succès dans un grand nombre d'activités et est aujourd'hui largement connue et reconnue. Elle convainc aussi par une culture et un humanisme où l'on sent l'influence de sa première éducation grecque et européenne. Guère contestable que son histoire n'est pas simplement celle d'une coach en développement personnel à la mode. Lecture intéressante et très actuelle. 